\title{
A case of adenoid cystic carcinoma of trachea: treatment complications and radiotherapy role
}

\author{
Aleksandra Piórek, MD, PhD', Sylwia Tabor, MDl, Piotr Jaśkiewicz, MD, PhD', Anetta Kasprowicz, MD², \\ Magdalena Zaborowska-Szmit, MD!, Kinga Winiarczyk, MD', Adam Płużański, MD, PhD', Prof. Dariusz Kowalski, MD, PhD', \\ Prof. Maciej Krzakowski, MD, PhDl \\ 'Lung Cancer and Chest Tumors Department, Maria Sklodowska-Curie National Research Institute of Oncology, Warsaw, Poland \\ ${ }^{2}$ Brachytherapy Department, Maria Sklodowska-Curie National Research Institute of Oncology, Warsaw, Poland
}

\begin{abstract}
Purpose: Adenoid cystic carcinoma (ACC) of trachea is a relatively rare malignant neoplasm, for which there is a lack of prospective clinical trials investigating treatment effectiveness. Most of the authors prefer surgical resection followed by post-operative radiation therapy in case of incomplete excision. There are no available prospective data on post-relapse treatment.

Case presentation: The current paper presents a case of tracheal ACC in a young woman, treated solely with surgical resection without radiotherapy due to postoperative neurological complications requiring additional diagnostics, management, and treatment. As a complication itself, spinal cord dysfunction after tracheal surgery is extremely rare, in which radical radiotherapy and brachytherapy were successfully administered after disease recurrence.

Conclusions: Lack of post-operative radiotherapy resulting from neurological complications could be a reason for ACC recurrence in our patient. Administration of radiotherapy after incomplete resection of recurrent disease may lead to long-term locoregional control.

J Contemp Brachytherapy 2021; 13, 5: 588-592 DOI: https://doi.org/10.5114/jcb.2021.109853
\end{abstract}

Key words: tracheal tumors, adenoid cystic carcinoma, radiotherapy, spinal cord dysfunction.

\section{Purpose}

Primary tracheal neoplasms are rare tumors. Their incidence is about 0.1 per 100,000 persons per year, representing approx. $0.2 \%$ of all respiratory tract tumors and $0.02 \%$ to $0.04 \%$ of all malignant tumors. About $90 \%$ of tracheal tumors in adults are malignant, compared with $10 \%$ to $30 \%$ of those in children [1]. Adenoid cystic carcinoma (ACC) is the second most common primary tracheal tumor after squamous cell carcinoma (SCC) [2, 3]. Rarity of this neoplasm results in an absence of prospective clinical trials investigating treatment effectiveness. Most of the authors prefer surgical resection, followed by post-operative radiation therapy in case of an incompleteness. There are no available prospective data on post-relapse treatment.

\section{Case report}

A 28-year-old woman diagnosed with tracheal ACC was presented to the Maria Sklodowska-Curie National Research Institute of Oncology in August 2012. The condition was diagnosed in 2008 when the patient was treated for dyspnea, which developed one month after undergoing a cesarean section. Diagnostic imaging re- vealed a mass measuring approximately $8-9 \mathrm{~cm}$, located below vocal cords on the right posterolateral wall of the trachea. Rigid bronchoscopy and electrocautery excisional biopsy of a $2 \mathrm{~cm}$ fragment of the tumor were performed urgently under general anesthesia due to severe dyspnea, resulting in a diagnosis of ACC and improvement of general condition. Subsequently, the patient was qualified for surgical treatment, and a tracheal sleeve resection and anastomosis were performed, with a positive margin of resection (R1) found on a pathology report. The patient's head was positioned anteriorly (chin-on-chest) after surgery. On the second day after surgery, she reported feeling weakness in her lower limbs. Magnetic resonance imaging (MRI) of the head and cervical spine showed no pathological findings. A neurological exam revealed moderate paresis, increased muscle tension, partial loss of superficial sensation in lower limbs, mild distal paresis with vivid tendon reflexes, and preserved superficial sensation in upper limbs. Analysis of cerebrospinal fluid (CSF) collected during lumbar puncture found no abnormalities. Physiotherapy improved the patient's clinical condition and resolved associated symptoms. The patient was closely oncologically monitored after surgery, and a follow-up bronchoscopy in July 2012 revealed two 
polypoid lesions located in lower part of the trachea and right main bronchus (7 and $2 \mathrm{~mm}$, respectively). A recurrence of ACC was confirmed via biopsies collected during bronchoscopy. Thoracic computed tomography (CT) found no evidence of other lesions. The patient was then referred to our department, where she was qualified for radiotherapy. A dose of 4,400 cGy/planning treatment volume (PTV) in fractions of 200 cGy/PTV was administered to the areas of recurrence (trachea, right main bronchus, and mediastinum), followed by a boost
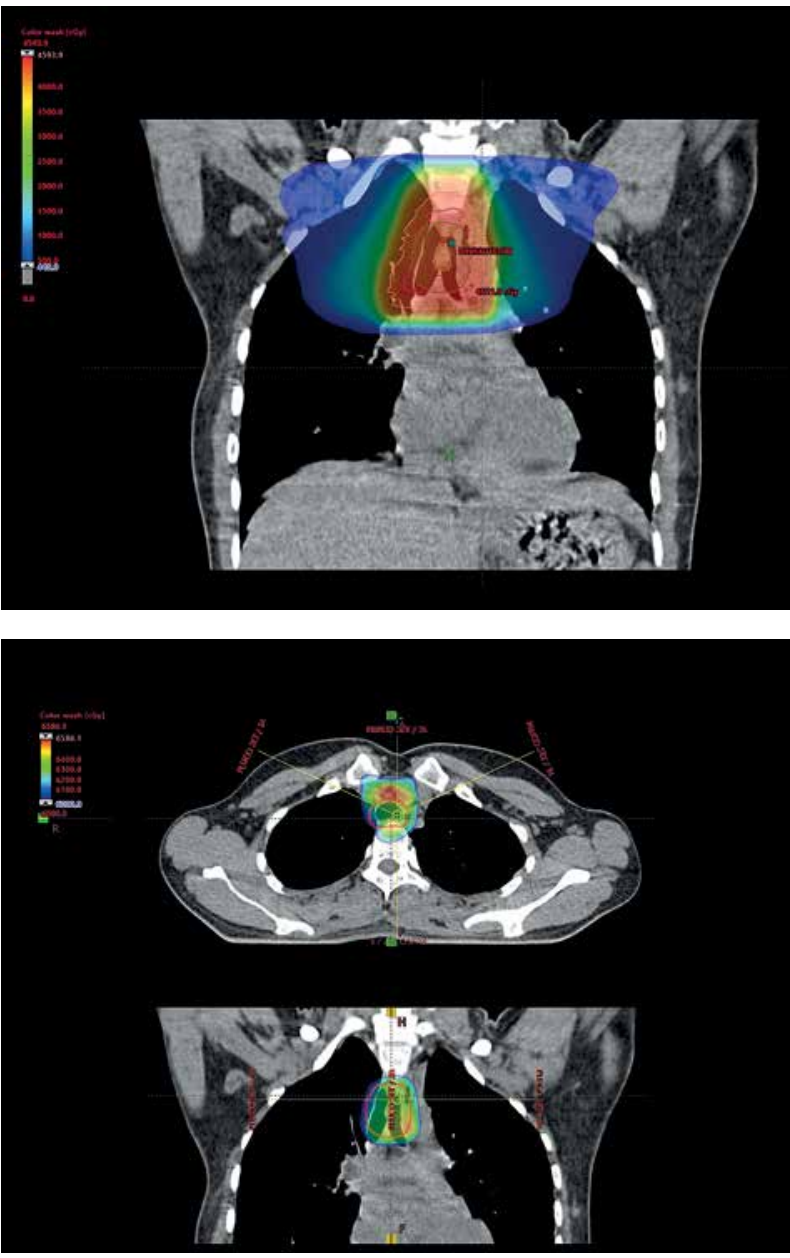

to a total dose of $6,400 \mathrm{cGy} / \mathrm{PTV}$ using standard fractionation. Radiotherapy planning was based on $6 \mathrm{MV}$ and $15 \mathrm{MV}$ photons using 3D conformal radiotherapy (3D-CRT) techniques. Lung volume receiving $\geq 20 \mathrm{~Gy}$ was $7.1 \%\left(V_{20}, 7.1 \%\right)$ and $\geq 5$ Gy was $29.6 \%\left(V_{5}, 29.6 \%\right)$. Mean lung dose was 5.6Gy and maximum spinal cord dose was $4.3 \mathrm{~Gy}$. Volume of the esophagus, which received $60 \mathrm{~Gy}$ was $17.9 \%$, and mean dose to the esophagus was 19.5Gy (Figures 1 and 2). Two weeks after completion of external beam radiotherapy, endoluminal high-dose-

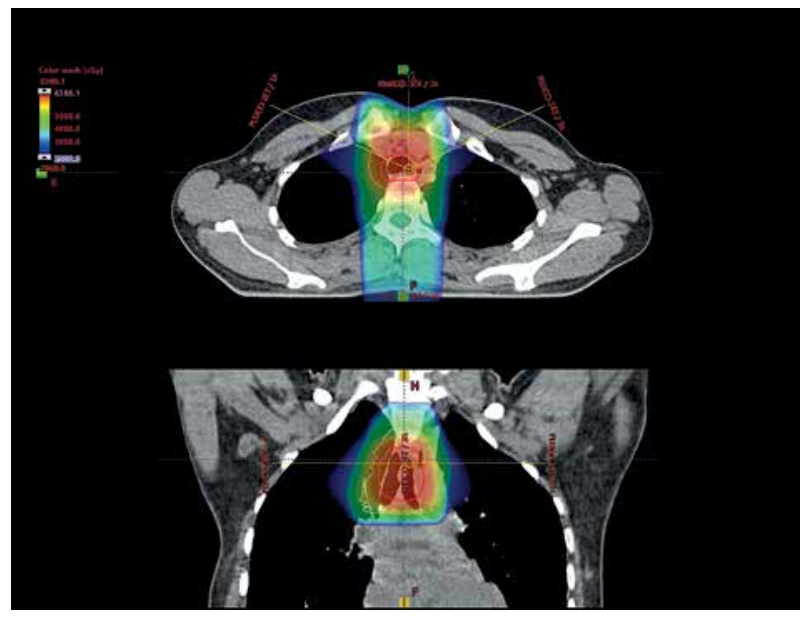

Fig. 1. Radiotherapy plan presented in computed tomography scans of the treated region with $3 \mathrm{D}$ reconstruction. Isodoses levels are presented in the dose color wash method from dose 1,300 cGy (blue) to 6,580 cGy (red)

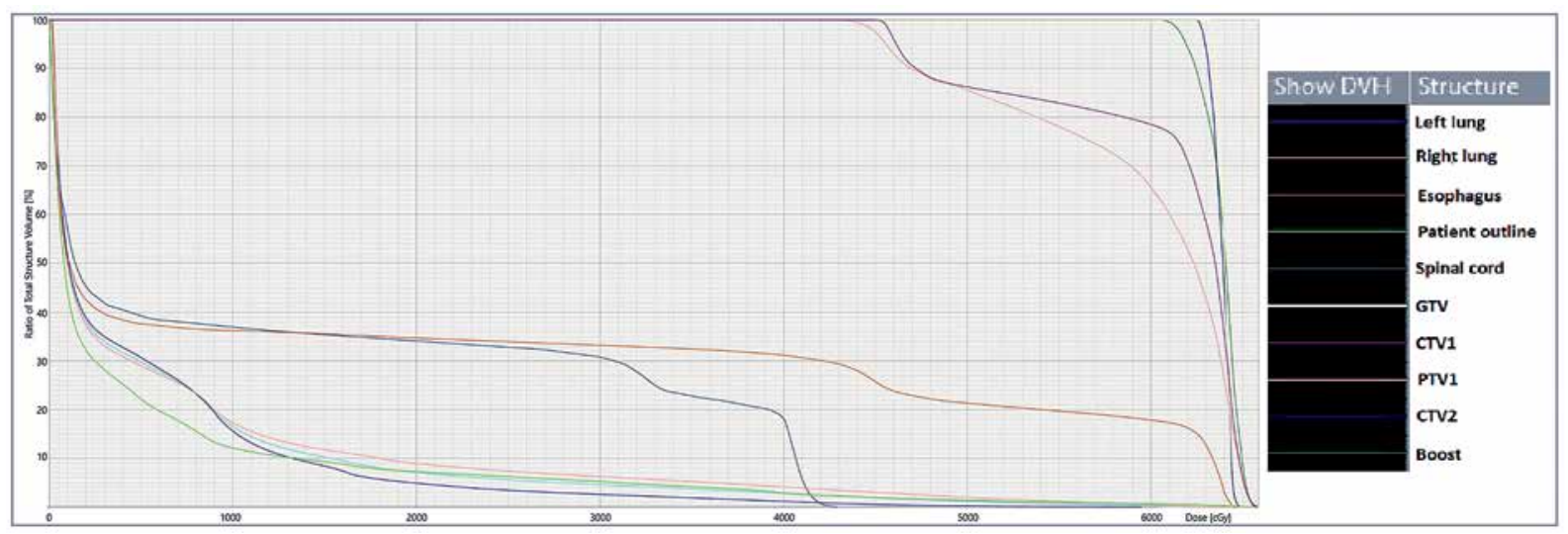

Fig. 2. Dose-volume histograms for the patient's treatment plan from Figure 1 
rate (HDR) brachytherapy was performed. Bronchoscopy was carried out after local anesthetic administration and revealed the presence of residual tumor tissue located on the main carina in both white light and autofluores- cence (Figure 3A, B). Fritz adjustable intralumenal applicator was used during the procedure (Figure 4A-C). Its design avoids administering a high mucosal dose by immobilizing and centering applicator within the lumen.
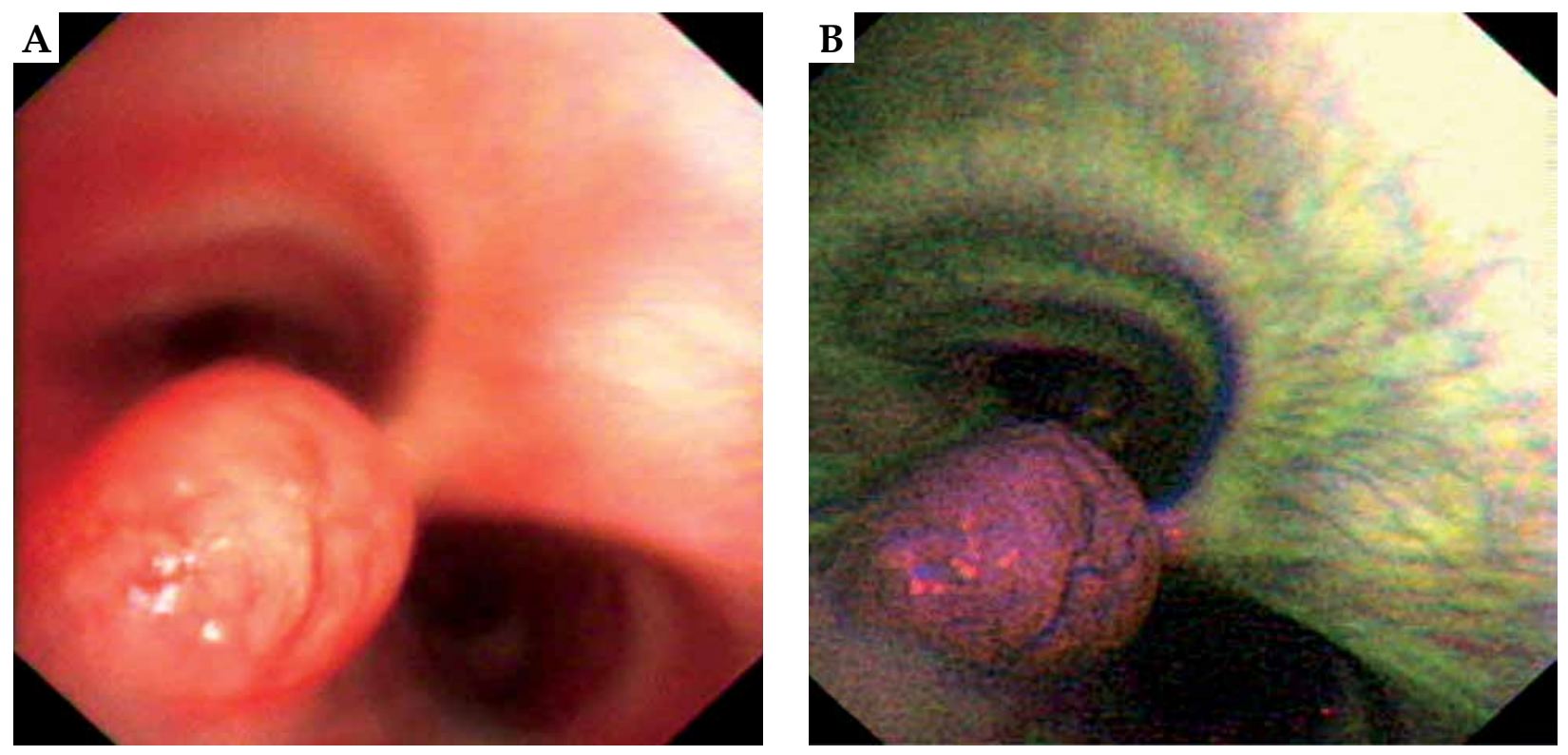

Fig. 3. The figures present images from video-bronchoscopy. A) White light video-bronchoscopy revealing a residual tumor located on main carina; B) Under autofluorescence imaging of video-bronchoscopy, the tumor showed reddish brown area with defined margin on normal mucosa appearing in green color
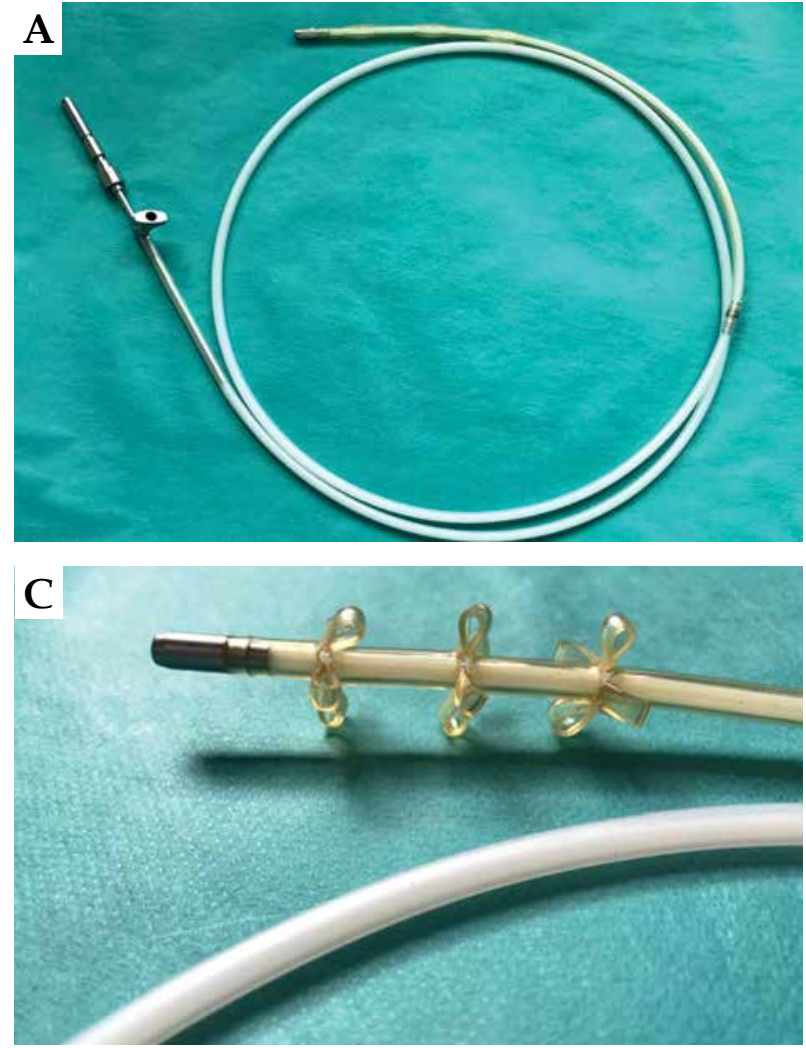

Fig. 4. A-C) Images of Fritz adjustable intralumenal applicator in different shots; D) Fluoroscopy with visible Fritz applicator
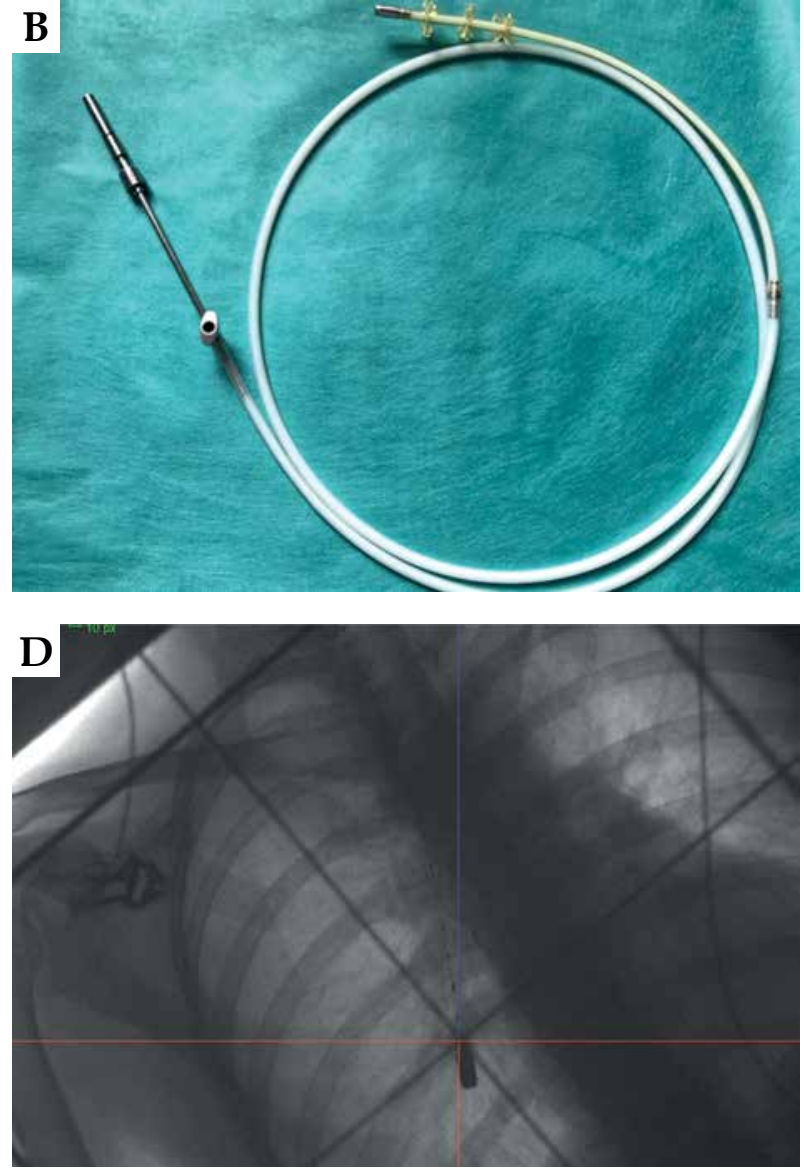
The applicator was inserted into the trachea near the tumor using a modified Seldinger technique and fixed in the right main bronchus. Orthogonal localization film was taken to ensure correct positioning of the applicator and to set a $6 \mathrm{~cm}$ target length (Figure 4D), which included residual tumor and $2 \mathrm{~cm}$ margins both proximally and distally. Two 5 Gy fractions were applied at one-week intervals prescribed at reference points one centimeter from the source. According to the Radiation Therapy Oncology Group (RTOG) acute radiation morbidity scoring criteria, the patient developed radiation-induced grade 2 esophagitis and was treated with analgesics and a pureed diet, resulting in a significant improvement. Radiotherapy was completed in October 2012, and since then, the patient remains in observation. Follow-up bronchoscopy and CT scans found no signs of a recurrence, with the most recent scan performed in October 2020. The patient stays in a very good overall condition, with ECOG $=0$.

\section{Discussion}

Adenoid cystic carcinoma arises from a minor salivary and serous glands presence within tracheal submucosa, and is morphologically similar to primary tumors of salivary glands [4]. ACC usually demonstrates exophytic growth leading to tracheal lumen narrowing [2], and is characterized by submucosal and perineural spread. Regional lymph node involvement or distant metastases are detected at the time of diagnosis in up to $10 \%$ of patients [2]. Some ACC are associated with slower rates of disease progression, with a five-year survival rate of about $90 \%$ and less than $40 \%$ survival at 15 years. More aggressive cases are associated with advanced-stage neoplasia at the time of diagnosis and more frequent incidence of recurrence and distant metastases. Distant or local recurrence can occur after a variable period of time [2]. ACC develops predominantly in the fourth and fifth decade of life, with similar frequency in men and women [2]. The etiology is unknown, but is not associated with smoking.

Radical surgical management is the treatment of choice whenever possible. The extent of resection depends on many factors, such as tumor size, location, and local extent [2]. The process of patient qualification for surgery should also include additional factors, including the amount of normal trachea that would remain after planned resection, any history of previous mediastinal surgeries or radiotherapy, age, and patient's physique [5]. Older patients with a short, stiff neck, are more likely to receive limited surgical resection in contrast to younger, lean, and tall patients. Importantly, this kind of surgery often requires particular patient's positioning, which must be modified throughout the procedure. Neck extension is also limited during post-operative care $[5,6]$. The most common complications of tracheal surgery include anastomotic leak (0.04-23.8\%), chyle leak (4.3-22\%), new recurrent laryngeal nerve paralysis $(0.04-55 \%)$, esophageal fistula (5-6\%), unplanned permanent tracheostomy $(4.3-50 \%)$, respiratory failure or prolonged intubation $(10 \%)$, tracheal stenosis $(5 \%)$, and wound infection $(1.4-11 \%)$ [7].

Until now, a rare event of cervical spinal cord disfunction was described in only some case reports, with one related to ACC [8-10]. Excessive neck flexion after surgery and sitting position resulting in low blood pressure appear to be the main causal factors. Reviewing the available literature, Windfuhr et al. presented possible etiologies, which must be considered when spinal cord disfunction is diagnosed [11]. According to the authors, an informed consent for surgery should include the possibility of such a rare complication, due to its unpredictability. Appropriate post-operative care consisting of daily neurological exams and avoidance of excessive neck flexion after surgery was also highlighted. In the presented case, the patient's head was positioned anteriorly during the post-operative period. Unfortunately, the detailed data regarding post-operative care, diagnostic processes, and treatment were limited due to main surgery having been performed in a district hospital. However, the consistency of clinical outcome and evidence coming from case reports allow us to suspect a diagnosis of cervical spinal cord disfunction.

The role of post-operative radiotherapy in ACC is unclear. Majority of publications recommend radiotherapy in cases of incomplete resection; however, this suggestion was not based on results of randomized prospective clinical trials [12-14]. Adjuvant radiation in a variety of dosages was used by Maziak et al. in 25 of 32 patients managed by primary resection. There was no statistically significant difference in survival between these 25 patients receiving adjuvant radiation and the seven patients who did not. Six of the seven patients, who had no adjuvant radiotherapy, accomplished a complete resection. The authors compared their results with observations reported in previous publications. They concluded that it appears logical to assume that adjuvant radiotherapy may be beneficial and likely to delay or even reduce the incidence of local recurrence within the airway, even though the role of adjuvant radiotherapy was impossible to evaluate with certainty [15]. The standard dose for adjuvant treatment is $60 \mathrm{~Gy}$ in fractions of $2 \mathrm{~Gy}$, given 5 times per week over 6 weeks [2]. In case of gross residual tumor mass, the dose should be increased to 68-70 Gy in fractions of 2 Gy [2]. Post-operative radiotherapy planning must be based on pre-surgical CT scans [16].

Radical radiotherapy alone is performed in patients who are not candidates for radical resection surgery due to medical contraindications or lack of consent. Adequately staged patients in good general condition are eligible for this kind of treatment. ACC exhibits low radiation sensitivity, and the required dose of irradiation to maintain local cancer control was estimated as $70 \mathrm{~Gy}$ (35 fractions over 7 weeks) $[2,16]$. Radiotherapy should be planned using conformal techniques, preferably intensity-modulated radiation therapy (IMRT) [16]. Endotracheal brachytherapy with 8-15 Gy may improve local control when applied after external-beam radiotherapy (60-68 Gy) [2]. Further research should be conducted to determine the maximum and optimum dose of endotracheal brachytherapy as a method, which can potentially increase the total dose when added to external beam radiotherapy [2].

Radiotherapy in tracheal cancer patients is well-tolerated in terms of toxicity. In a retrospective study includ- 
ing 48 radiotherapy patients, most of them (31 patients) did not report any side effects. In the rest of analyzed group, dysphagia was the most frequently reported adverse event (8 patients), followed by dyspnea (3 patients), oedema ( 2 patients), and pain ( 2 patients). One person suffered from deterioration of their general condition. In most cases, toxicity was evaluated as low-grade. Amongst 8 patients receiving brachytherapy, hemorrhage was reported only once. The rest of patients did not develop any clinical signs of toxicity after this treatment [17].

Lack of adjuvant radiotherapy due to post-operative neurological complications was a probable reason for ACC recurrence in our patient. Administration of external beam radiotherapy, followed by brachytherapy boost after incomplete resection of the recurrent disease, may lead to long-term locoregional control.

\section{Disclosure}

The authors report no conflict of interest.

\section{References}

1. Junker K. Pathology of tracheal tumors. Thorac Surg Clin 2014; 24: 7-11.

2. Macchiarini P. Primary tracheal tumours. Lancet Oncol 2006; 7: 83-91.

3. Urdaneta AI, Yu JB, Wilson LD. Population based cancer registry analysis of primary tracheal carcinoma. Am J Clin Oncol 2011; 34: 32-37.

4. Wu CC, Shepard JA. Tracheal and airway neoplasms. Semin Roentgenol 2013; 48: 354-364.

5. Rea F, Zuin A. Tracheal resection and reconstruction for malignant disease. J Thorac Dis 2016; 8 (Suppl 2): S148-152.

6. Afshar N, English DR, Thursfield V et al. Differences in cancer survival by sex: a population-based study using cancer registry data. Cancer Causes Control 2018; 29: 1059-1069.

7. Madariaga ML, Gaissert HA. Secondary tracheal tumors: a systematic review. Ann Cardiothorac Surg 2018; 7: 183-196.

8. Dominguez J, Rivas JJ, Lobato RD et al. Irreversible tetraplegia after tracheal resection. Ann Thorac Surg 1996; 62: 278-280.

9. Silver JR. Paraplegia as a result of tracheal resection in a 17-year-old male. Spinal Cord 2007; 45: 576-578.

10. Pitz CC, Duurkens VA, Goossens DJ et al. Tetraplegia after a tracheal resection procedure. Chest 1994; 106: 1264-1265.

11. Windfuhr JP, Dulks A. Spinal cord infarction following cricotracheal resection. Int J Pediatr Otorhinolaryngol 2010; 74: 1085-1088.

12. Shimizu J, Oda M, Matsumoto I et al. Clinicopathological study of surgically treated cases of tracheobronchial adenoid cystic carcinoma. Gen Thorac Cardiovasc Surg 2010; 58: 82-86.

13. Hu MM, Hu Y, He JB et al. Primary adenoid cystic carcinoma of the lung: Clinicopathological features, treatment and results. Oncol Lett 2015; 9: 1475-1481.

14. Yang H, Yao F, Tantai J et al. Resected tracheal adenoid cystic carcinoma: improvements in outcome at a single institution. Ann Thorac Surg 2016; 101: 294-300.

15. Maziak DE, Todd TR, Keshavjee SH et al. Adenoid cystic carcinoma of the airway: thirty-two-year experience. J Thorac Cardiovasc Surg 1996; 112: 1522-1531; discussion 1531-1522.

16. Pawlewicz K, Szutkowski Z, Kawecki A. Recurrence of adenoid cystic carcinoma of the trachea treated with radical radiotherapy: A case report. Oncol Lett 2018; 15: 3890-3894.

17. Napieralska A, Miszczyk L, Blamek S. Tracheal cancer treatment results, prognostic factors and incidence of other neoplasms. Radiol Oncol 2016; 50: 409-417. 